\title{
CALIBRATION OF TUFFAK POLYCARBONATE TRACK DETECTOR FOR IDENTIFICATION OF RELATIVISTIC NUCLEI
}

\author{
Shl.Lun Guo ${ }^{\dagger}$, J. Drach* ${ }^{*}$, P. B. Price* ${ }^{*}$, M. H. Salamon*, M. L. Tincknell ${ }^{*}$ \\ Department of Physics, University of California, Berkeley, California 94720, U.S.A. \\ S. P. AHLEN \\ Department of Physics, Indiana University, Bloomington, Indiana 47405, U.S.A. \\ and \\ G. TARLÉ \\ Department of Physics, Randall Laboratory \\ University of Michigan, Ann Arbor, Michigan 48109, U.S.A.
}

\section{(Received 8 October 1984)}

\begin{abstract}
We discuss response of Tuffak polycarbonate to relativistic heavy nuclei using two methods, measurement of the minor axis diameter and of the length of the track cone, to determine charge resolution. At $Z=92\left(0.95 \mathrm{GeV} / \mathrm{u}{ }^{238} \mathrm{U}\right)$ both methods give about $0.9 e$ charge resolution for a single cone measurement. Multiple cone measurements along the ion's trajectory have yielded a charge resolution $\sigma_{\mathrm{z}} \leqslant 0.25 e$ ( 16 cones) when stripping foils $(\mathrm{Cu})$ are interleaved between plastic shects to minimize shect-to-sheet charge state correlations. As the charge of the incident ion decreases to $Z \approx 52-57$, the single-cone charge resolution improves $\left(\sigma_{z} \sim 0.29 e\right)$. The angular response of Tuffak is fairly constant for zenith angles of incidence from $0^{\circ}$ to $48^{\circ}$. Range measurements of stopping relativistic ${ }^{238} \mathrm{U}$ in Tuffak deviatc by $\sim 5 \%$ from that predicted by the Bethe-Bloch formula, as expected from recent relativistic calculations. We conclude that Tuffak is an excellent track detector for identification of nuclear charges of relativistic heavy nuclei with $50 \leq Z \leq 92$.
\end{abstract}

\section{INTRODUCTION}

IN THE study of ultra-heavy cosmic rays and relativistic heavy ion nuclear interactions, one needs a series of nuclear track detectors with high charge resolution for relativistic heavy nuclei. In a previous study, Price et al. (1983) and Salamon et al. (1984) have shown that CR-39 is an excellent nuclear track detector with high charge resolution for relativistic nuclei of $10 \leq Z \leq 60$. The next important task is to find detector materials with high charge resolution for relativistic nuclei of $60 \leq Z \leq 92$ or even heavier. For this purpose, silica glass, Melinex, Lexan, CR-39, Tuffak and BPADC (bisphenol-A diallyl carbonate) (Ahlen et al., 1984) have been tested. As a result of these tests, study has focussed on Tuffak polycarbonate plastic because of its superiority over others for identification of heavier relativistic nuclei (O'Sullivan and Thompson, 1981).

Since track length as well as cone diameter measurements are used in cosmic ray and relativistic nuclear physics studics empioying plastic detectors, we examined the properties of Tuffak making use of both measurement techniques. In this study we examine the range-energy relation of relativistic uranium in Tuffak, the reduced etch rate versus $Z^{*} / \beta$, the reduced etch rate versus residual range, the charge resolution obtainable with track length and track diameter measurements, charge resolution improvement for actinide nuclei by using a stripping medium, and the angular response of Tuffak for different angles of incidence.

\footnotetext{
*Also Space Sciences Laboratory, University of California, Berkeley, CA 94720, U.S.A.

+Also Lawrence Berkeley Laboratory, University of California, Berkeley, CA 94720, U.S.A.

Present address: Institute of Atomic Energy, Academia Sinica, P.O. Box 275(2), Beijing, China.
} 


\section{RANGE-ENERGY RELATION FOR RELATIVISTIC ${ }^{238} \mathrm{U}$ IONS IN TUFFAK}

Ahlen and Tarle have shown that $955 \mathrm{MeV} / \mathrm{u}$ ${ }^{238} \mathrm{U}$ in copper has an obvious range deficit (Ahlen and Tarlé, 1983) from the standard Bethe-Bloch formula (Ahlen, 1980). They showed that the discrepancy is of significance for high energy astrophysics experiments. In order to see if the range of relativistic ${ }^{237} \mathrm{U}$ ions in plastic track detectors has a range deficit, we started our study by measuring the range of relativistic ${ }^{238} \mathrm{U}$ ions in Tuffak.

A stack composed of 369 sheets of Tuffak was exposed to a ${ }^{238} \mathrm{U}$ beam of $955 \mathrm{MeV} / \mathrm{u}$ at a zenith angle of $\sim 10^{\circ}$ at Lawrence Berkeley Laboratory's Bevalac. Each sheet of the stack had an area $7.2 \times$ $7.2 \mathrm{~cm}^{2}$ and a thickness $\sim 0.265 \mathrm{~mm}$. The Tuffak stack was thick enough to stop the ${ }^{238} \mathrm{U}$ ions.

One out of every ten sheets was first etched at $40^{\circ} \mathrm{C}$ in $6.25 \mathrm{~N} \mathrm{NaOH}$ and $0.05 \%$ Dowfax surfactant and saturated with Lexan etch products. The etching time was changed from sheet to sheet to ensure that the tracks in each sheet could be seen easily under the microscope. All sheets around the stopping point of the ${ }^{238} \mathrm{U}$ ions were then etched. The stopping points of about $120{ }^{238} \mathrm{U}$ ions were then measured with an optical microscope. Figure I shows the histogram of stopping points of ${ }^{238} \mathrm{U}$ ions

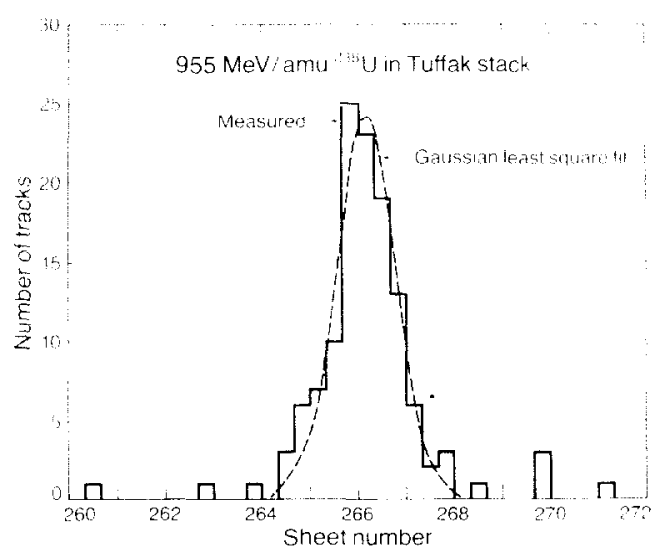

FIG. 1. Histogram of stopping points of ${ }^{23 \mathrm{H}} \mathrm{U}$ ions in Tuffak stack. Standard deviation of range of $955 \mathrm{MeV} / \mathrm{u}$ ${ }^{238} \mathrm{U}$ in Tuffak stack is 0.60 sheets. The range of ${ }^{238} \mathrm{U}$ in Tuffak is $5 \%$ shorter than that calculated from standard Bethe-Bloch formula. in the Tuffak stack. The standard deviation of the range histogram is 0.60 sheet of Tuffak. The range straggling was found to be $0.23 \%$, which is consistent with that expected for wry heary projectiles.

The measured range of ${ }^{3} \mathrm{U}$ ions in I uffak stact is about $5 \%$ shorter than that calculated by the Bethe-Bloch stopping power formula and is consistent with that calculated after inclusion of the Mott cross section and the relativistic Bloch correction (Ahlen, 1982) in the stopping power formula

In the following analysis we use the result of the range measurements to calculate parameters such as ion velocity in our study of charge resolution.

\section{REDUCED ETCH RATE VERSUS $Z^{*} / \beta$}

The same Tuffak stack used tor range measure ments was used to get a relation between the reduced etch rate $S$ and $Z^{*} / \beta$, where $S=V_{r} V_{i}$. $V_{I}$ is etch rate along particle track: $V_{C_{i}}$ is bulk etch rate: $Z^{*}$ is the effective charge of the incident heavy nuclei (Ahlen, 1980); Pierce and Blann. 1968):

$$
X^{\prime}=Z\left(1+\mathrm{C}^{\circ}\right.
$$

where $Z$ is atomic number of the incident nucleus and $\beta$ is its velocity (in units of speed of light) as determined by its residual range in the stack. In the interval of $Z^{*} / \beta$ from $\sim 105$ to $\sim 170$, we selected 1.3 sheets of Tuffak for etch rate measurements. Etching was performed with the same conditions as those in the range measurements. The etching time varied from 12.6 to $84 \mathrm{~h}$ so that the total length of the two cones of each Tuffak sheet (one per side) equaled $90 \%$ of the path length of the ${ }^{23 \pi} \mathrm{U}$ ion $\mathrm{In}$ the sheet; this minimized relative track length errors.

The track length was measured under 5.3 (oil) $x$ 10 magnification of a Leitz Largefield Metallographic Microscope (METALLOPLAN) with a linear displacement transducer for accurate depth measurement. The deviation of track length measurements was found to be less than $0.4 \mu \mathrm{m}$ in the interval of track lengths from 20 to $70 \mu \mathrm{m}$.

The reduced etch rate $S$ was calculated from the track geometry using the formula (Fleischer $e t a l$., 1975) 
$S=\left[\sin \left\{\frac{1}{2}\left[-\arctan \left(\frac{Q}{a+p}\right)+\arctan \left(\frac{Q}{p}\right)\right]\right\}\right]^{-1}$

where $a, p$ and $Q$ are shown in Fig. 2 .

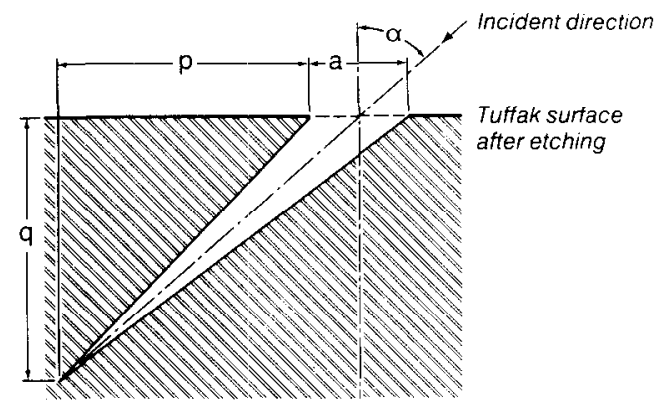

FIG. 2. Track geometry of a particle with incidence angle $\alpha$.

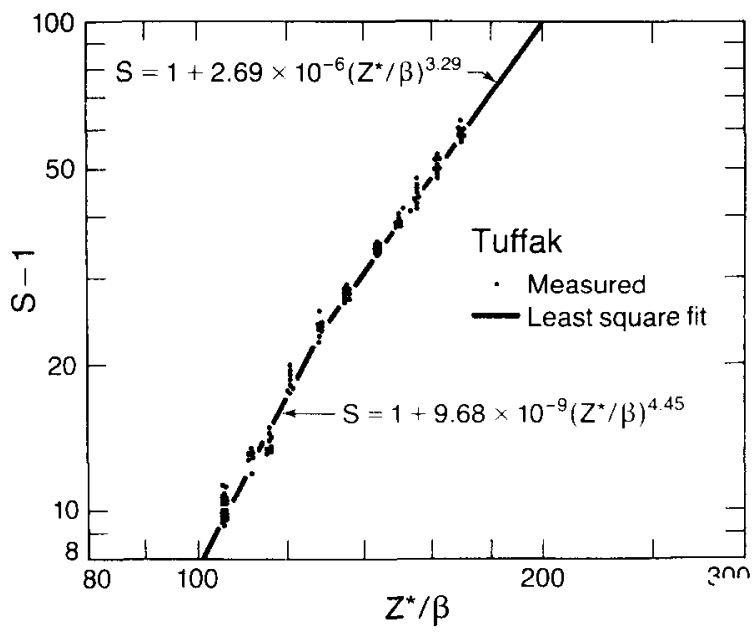

FIg. 3. Relation of reduced etch rate $S$ to $Z^{*} / \beta$ in Tuifak. Least square fit to two straight lines:

$$
\begin{array}{ll}
S=1+9.68 \times 10^{-9}\left(Z^{*} / \beta\right)^{4.45} & \left(105 \leq Z^{*} / \beta \leq 130\right) \\
S-1+2.69 \times 10^{-6}\left(Z^{*} / \beta\right)^{3.29} & \left(130 \leq Z^{*} / \beta \leq 180\right) .
\end{array}
$$

A plot of reduced etch rate $S$ versus $Z^{*} / \beta$ is shown in Fig. 3. Here we use two straight lines in bi-logarithmic coordinates to fit the $S$ data:

$$
S=1+9.68 \times 10^{-9}\left(Z^{*} / \beta\right)^{4.45} \quad 105 \leq Z^{*} / \beta \leq 130
$$

$S=1+2.69 \times 10^{-6}\left(Z^{*} / \beta\right)^{3.29} \quad 130 \leq Z^{*} / \beta \leq 180$.

\section{REDUCED ETCH RATE VERSUS RESIDUAL RANGE OF ${ }^{238}$ U IN TUFFAK}

From track length and residual measurements of ${ }^{238} \mathrm{U}$ tracks in Tuffak, the relation between reduced etch rate $S$ and residual range $R_{0}$ of ${ }^{238} \mathrm{U}$ in Tuffak can be obtained. The relation is shown in Fig. 4. All ${ }^{238} \mathrm{U}$ data which are below $955 \mathrm{MeV} / \mathrm{u}$ fall approximately on a straight line in bi-logarithmic coordinates. This line can be expressed by the formula $S=42.2{R_{0}}^{-0.63}$.

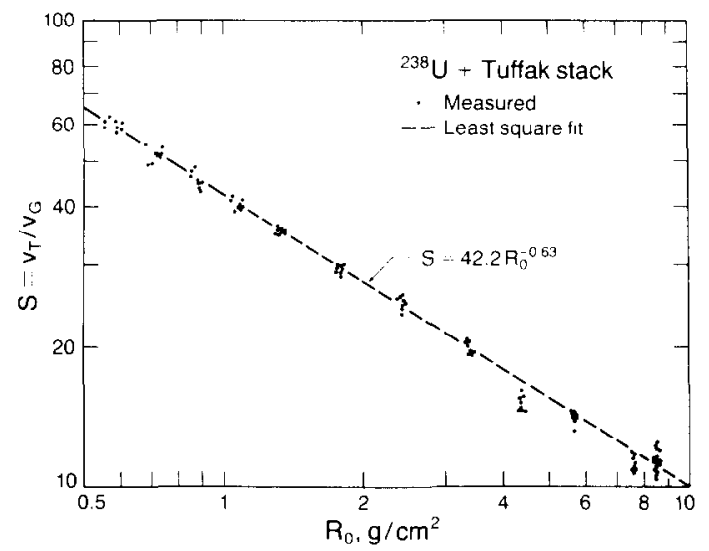

FIg. 4. Relations of reduced etch rate $S$ to residual range $R_{0}$ of ${ }^{238} \mathrm{U}$ in Tuffak. Straight line is a least square fit to measured date.

\section{CHARGE RESOLUTION OF TUFFAK FOR TRACK LENGTH MEASUREMENTS}

In the track length measurements described in Section 3, nine tracks of nine ${ }^{238} \mathrm{U}$ ions were followed through the stack. One of them suffered a nuclear interaction midway through the stack. The other eight ions' values of reduced etch rate in each sheet were used to calculate the standard deviation $\sigma_{s}$ of reduced etch rate for each sheet. The charge resolution $\sigma_{z}$ follows from

$$
\sigma_{z}=\sigma_{s} /(\partial S / \partial z)=\frac{\sigma_{s} \beta}{a b\left(Z^{*} / \beta\right)^{b-1}}
$$

where

$a=9.68 \times 10^{-9}, b=4.45$ for $105 \leq Z^{*} / \beta \leq 130$;

$a=2.69 \times 10^{-6}, b=3.29$ for $130 \leq Z^{*} / \beta \leq 170$; 
and $Z=92$.

The calculated charge resolution of Tuffak for ${ }^{2.3 \times} \mathrm{U}$ ions is shown in Fig. 5. The average value of charge resolution of Tuffak for ${ }^{23 *} \mathrm{U}$ in the energy region from $400 \mathrm{MeV} / \mathrm{u}$ to $955 \mathrm{MeV} / \mathrm{u}$ is about 0.89 e for a single track length measurement. Below $400 \mathrm{MeV} / \mathrm{u}$. the charge resolution has a larges fluctuation, but the average value is still below $0.9 e$.

\section{CHARGE RESOLUTION OF 'TUFFAK FOR TRACK DIAMETER MEASUREMENTS}

Another stack of Tuffak was perpendicularly exposed to ${ }^{238} \mathrm{U}$ ions of $962 \mathrm{MeV} / \mathrm{u}$ at the Bevalac After etching in the sante conditions as described in Section 2, track diameters in the top sheet werk measured in reflected light with a Leitz. Ortholux microscope coupled to a Compumetric AMS 100 system. The width of the track diameter distribution is a measure of $\sigma_{2}$. from which the charge resolution of $962 \mathrm{MeV} \mathrm{u}^{2.3 \times} \mathrm{U}$ in Tuffak is obtained. being $\sigma_{z}=0.9 e$ for single track diameter measure ment, almest the same value as we obtained from single track length medsurements. This datum is shown as a square in Fig. 5 .

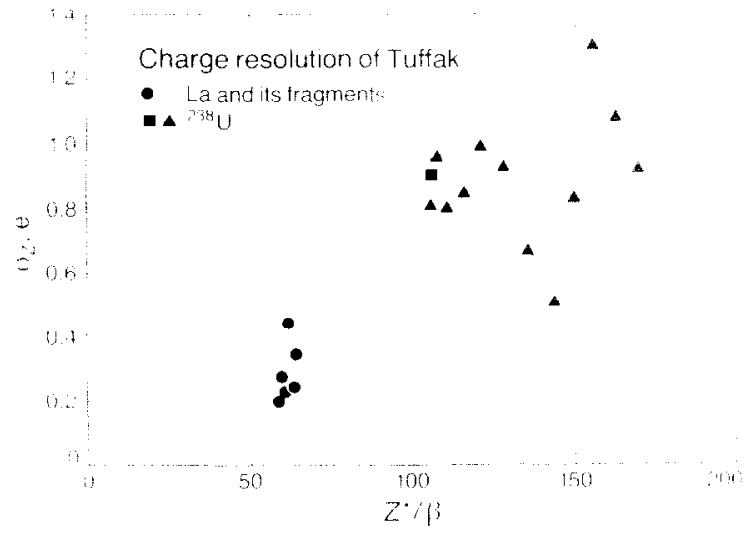

FK. 5. Charge resolution ol lulfak an at function of $/ \%$ of incident particles. ${ }^{23 \times} \mathrm{U}$ tracks were measured by both track length method $(\boldsymbol{\Delta})$ and track diameter method

La and its fragments were measured by track diameter method
A third stack composed of luftak. polyethylens (to) enhance nuclear interaction fragment production) and $\mathrm{Pb}$ (to increase ion slowing) was exposed (1) 1.25 GeViu ${ }^{1.45} 1$ at at the Bevalac. Several sheet of Tuffak were then etched in the same solution mentioned above for 239. $\mathrm{h}$ h. The track diameter from midstack were measured with the Compumetric system. A histogram of the trach diameter distribution is shown in Fig. fi. This distribution was ubtained by velecting $\$ 25$ cones for measurement an a single sheet surface, then following the tracks through 3 additional surfaces and averaging the diameters for each ion, the fragments diameter widths diminist: as 1 In where $n$ is the number of independent cone meatsurements for a given ion. The peak on the right side of the histogram corresponds to incident la ions. The whers correspond to fragments of $I$ a produced from nuckeal interactions with nuclei of the detector stack. The incident Lal peak in the histogram is reduced in height relative to the sther peaks because : diameter cut wats applicd during cons selection in the first surface to favor welection of fragmente 6 total of 977 . Tracks of particles which interacted in the region from the lirst 6 the last meatsured surfaces were climinated using the criterion that the track diameter difference $D$ between two conti guous cones be kes than 24 um lortering al

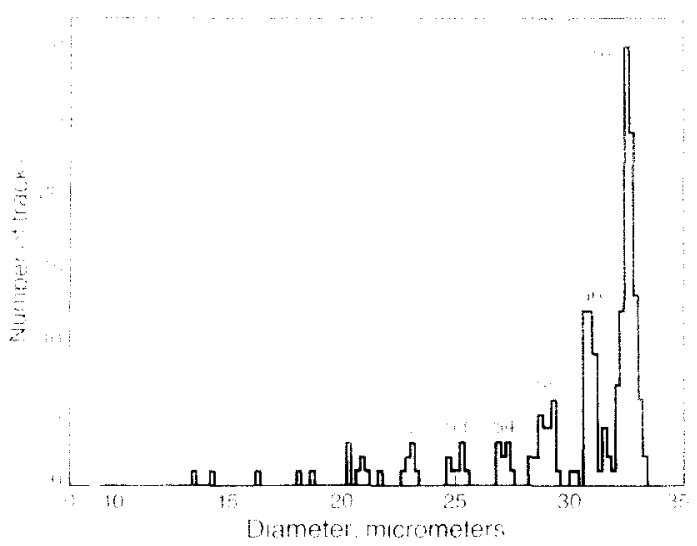

Fic. o. Track diameler distribution of refativestic Lat and its fragments (4 track cone measurement). If $\therefore I$ ) $\ldots . .4$ $\mu \mathrm{m}$, the tracks were climinated an interated coents, the

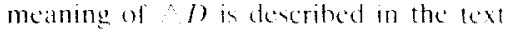


improves charge resolution at the expense of statistics.

The charge resolution of $\mathrm{La}$ and its fragments was obtained from the peak widths and the separation between the individual peaks. The results are shown by solid circles in Fig. 5. The average value of charge resolution of Tuffak for $52 \leq Z \leq 57$ is about $\sigma_{z}=0.29 e$ for a single track diameter measurement. Thus, Tuffak has a very good charge resolution for $Z$ as low as 52 , being much better in this region than CR-39.

\section{CHARGE RESOLUTION OF TUFFAK INTERLEAVED WITH STRIPPING FOILS}

As mentioned above, from a single track diameter measurement a charge resolution of $0.9 e$ can be achieved for $\sim 1 \mathrm{GeV} / \mathrm{u}{ }^{238} \mathrm{U}$. In order to get

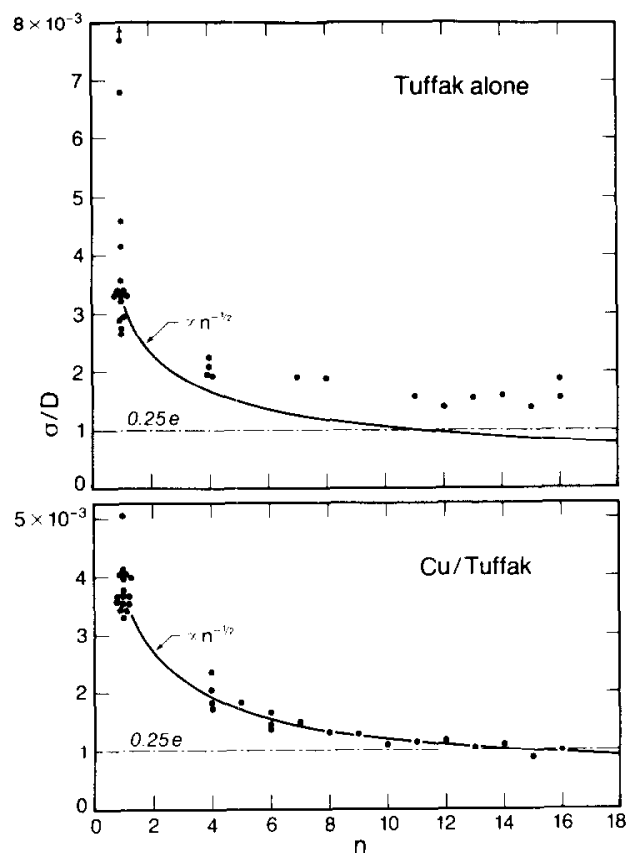

FIG. 7. Improvement of charge resolution of Tuffak as measuring $n$ cones successively along the same trajectory of ${ }^{238} \mathrm{U}$ ions. For $n>1$, the charge resolution of pure Tuffak stack (top figure) is poorer than that of Cu-Tuffak stack (bottom figure) which follows a factor of $1 / \sqrt{n}$. better charge resolution, many successive track cone diameters along the same ${ }^{238} \mathrm{U}$ trajectory were measured. (At relativistic energies, $Z^{*} / \beta$ values for adjacent sheets are almost the same.) The charge resolution obtained as a function of the number $n$ of successive track cone diameters measured is shown in Fig. 7. This particular work has been published previously, (Salamon et al., 1984) so we restrict our discussion to a couple of salient features: as seen in the figure, although $\sigma_{z}$ decreases as $n$ increases for a pure Tuffak stack, it does not fall as rapidly as $1 / \sqrt{n}$. Salamon et al. (1984) have explained this as being due to the statistics of electron capture and loss by the slowing uranium ion; charge state correlations from sheet to sheet destroy the statistical independence required for a $1 / \sqrt{n}$ falloff in charge width. Introduction of $\mathrm{Cu}$ stripper foils between each Tuffak sheet restores statistical independence by 'reshuffling' the charge state distribution, and it is seen in Fig. 7 that a charge resolution $\sigma_{z}=0.25 e$ has been achieved for $1 \mathrm{GeV} / \mathrm{u}{ }^{238} \mathrm{U}$ ions in Tuffak with $n=16$ cone diameter measurements.

\section{ANGULAR RESPONSE OF TUFFAK FOR DIFFERENT INCIDENT ANGLES}

Knowing the angular response of nuclear detector is very important for detecting particle sources with varying incidence angles. A fifth stack of Tuffak was exposed to $955 \mathrm{MeV} / \mathrm{u}{ }^{238} \mathrm{U}$ beam at four incident angles $(\alpha): \sim 0^{\circ}, 15.6^{\circ}, 31.9^{\circ}$ and $48.0^{\circ}$. After etching, track lengths of 9 ions for each angle were measured, yielding an average reduced etch rate $S$ value for each angle. The resulting angular

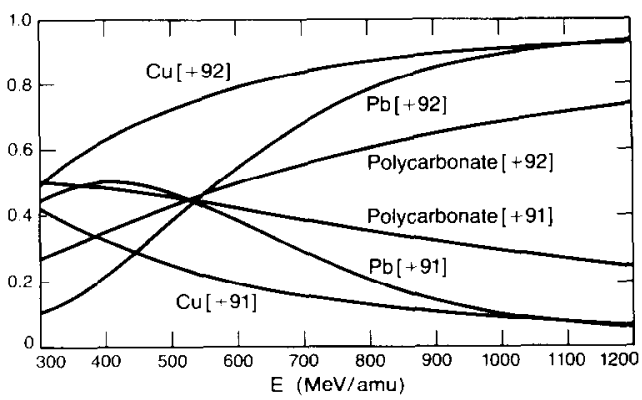

FIG. 8. Charge state fraction $[+92 e]$ and $[+91 e]$ of ${ }^{2.38} \mathrm{U}$ in Tuffak, $\mathrm{Cu}$ and $\mathrm{Pb}$ as a function of energy. 
response of Tuffak is shown in Fig. 10, and is fairly isotropic from $\alpha=0^{\circ}$ to at least $\alpha=48.0^{\circ}$. This property is important for studies of ultraheavy cosmic rays since angular correction factors are unnecessary.

\section{SUMMARY}

The study of charge resolution of Tuffak polycarbonate plastic shows that it is an excellent detector for identification of relativistic nuclei of 50 $\leq Z \leq 92$. When identifying very heavy nuclei the Mott cross section and relativistic Bloch correction

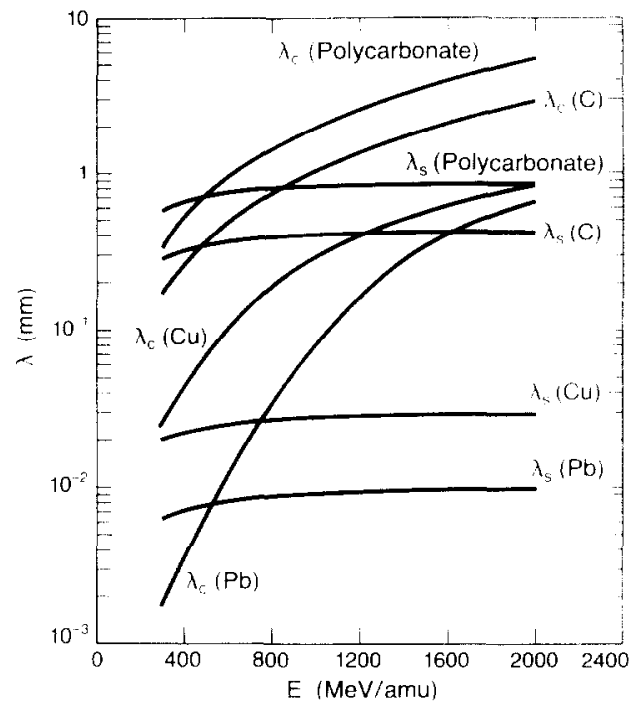

FIG. 9. Electron capture and stripping interaction lengths (in $\mathrm{mm}$ ) of ${ }^{238} \mathrm{U}$ in Tuffak, C, $\mathrm{Cu}$, and $\mathrm{Pb}$ as a function of energy.

must be employed in range and energy calculations Either track lengths or track diameters can be used to scan heavy nuclear tracks; both methods can achieve a charge resolution $\sigma_{z} \simeq 0.9 e$ for relativistic ${ }^{238} \mathrm{U}$ for a single track cone measurement. For lighter nuclei around $\mathrm{La}$, the charge resolution can reach $\sigma_{z} \sim 0.29 e$ for a single track diameter measurement. Stripping materials (Cu foils) interleaved with Tuffak can improve the charge resolution by removing sheet to sheet charge stack correlations, validating the $1 / \sqrt{n}$ law for relativistic actinides. A charge resolution $\sigma_{\mathrm{s}} \leq 0.25 \mathrm{e}$ for

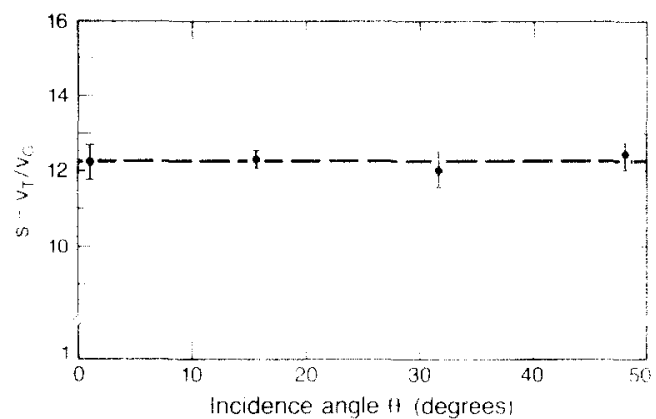

Fic; 10. Angular response of luffat at diflerent incident angles to 940 MeVa

relativistic ${ }^{2 i x} \mathrm{U}$ has thus been obtained. We ate sure that $\sigma_{z} \leq$ (). le is easy to reach in the region of $50<Z \leq 60$.

Acknowledgements-We are indebted to M Solarz for ho technical assistance in part of this work. Thanks are due to the staff at the Bevalac, LBL. especially Hank Crawford, for their help in exposure of the samples. Shi-Lun Guo thanks Prof. P. B. Price and all members of this group, especially Judy Blait. For hospitality and friendly help at Berkeley.

This work was supported on NASA crant NGR of $1913-376$

\section{REFERENCES}

Ahlen S. P. (1980) Rev. Mod. Phys. 52. 121

Ahlen S. P. (1982) Phys. Re't. A 25, 1856.

Ahlen S. P. and Tarle G. (1983) Phys Rel. Le'll so. 1110 .

Ahlen S. P., Coan I. E.. Drach J., Shi-Lun Guo, Price P. B., Salamon M. H. . Tarlé G. and Tincknell M. I. (1984) Nucl. Tracks Rad. Meas. 8.571

Fleischer R. L.. Price P. B. and Walker R. M. (1975) Nuclear Tracks in Solids: Principles and Applications. University of California Press. Berkeley.

O'Sullivan D. and Thompson A. (1981) Proc. / th Inter Conf. on Solid State Nuclear Track Detectom. Bristol. England. p. 85

Pierce T. E. and Blann M. (1968) Phys. Rer. 173, 390.

Price P. B., Tincknell M. L.. Tarlé G.. Ahlen S. P. Frankel K. A. and Perlmutter S. (1983) Phys. Re'. Lett. $50,566$.

Salamon M. H. Drach J., Shi-Lun Guo, Price P. B. Tarlé G. and Ahlen S. P. (1984) Nucl. Instrum. Meth. 224, 217.

Salamon M. H., Price P. B., Tincknell M. L.. Shi-Lun Guo and Tarlé G. (1984) Nuct Instrum. Meth. in press 